\title{
Orally Administered Semaglutide Versus GLP-1 RAs in Patients with Type 2 Diabetes Previously Receiving 1-2 Oral Antidiabetics: Systematic Review and Network Meta-Analysis
}

\author{
Solomon Nuhoho (D) - Jatin Gupta - Brian Bekker Hansen • \\ Mary Fletcher-Louis · Tam Dang-Tan · Abby Paine
}

Received: August 30, 2019 / Published online: October 10, 2019

(C) The Author(s) 2019

\section{ABSTRACT}

Introduction: Orally administered semaglutide is the first glucagon-like peptide 1 receptor agonist (GLP-1 RA) for oral administration. As head-to-head trials assessing orally administered semaglutide as an add-on to 1-2 oral antidiabetic drugs (OADs) vs other GLP-1 RAs are limited, a network meta-analysis (NMA) was performed to assess the relative efficacy and safety of orally administered semaglutide $14 \mathrm{mg}$ once-daily (QD) vs injectable GLP-1 RAs in

Enhanced Digital Features To view enhanced digital features for this article go to https://doi.org/10.6084/ m9.figshare.9901934.

Electronic Supplementary Material The online version of this article (https://doi.org/10.1007/s13300019-00706-y) contains supplementary material, which is available to authorized users.

S. Nuhoho $(\varangle) \cdot$ B. B. Hansen

Novo Nordisk A/S, Søborg, Denmark

e-mail: snuh@novonordisk.com

J. Gupta

DRG Abacus, Gurgaon, India

M. Fletcher-Louis

DRG Abacus, Bicester, Oxfordshire, UK

T. Dang-Tan

Novo Nordisk Inc., Plainsboro, NJ, USA

A. Paine

Zedediah Consulting, Berkshire, UK patients with type 2 diabetes inadequately controlled on 1-2 OADs.

Methods: A systematic literature review was conducted to identify randomised controlled trials of GLP-1 RAs in patients inadequately controlled on 1-2 OADs. Data at $26 \pm 4$ weeks were extracted for efficacy and safety outcomes feasible for the NMA: change from baseline in glycated haemoglobin $\left(\mathrm{HbA}_{1 \mathrm{c}}\right)$, weight, $\mathrm{HbA}_{1 \mathrm{c}}$ target levels $(<7.0 \%$ and $\leq 6.5 \%)$, blood pressure, and any gastrointestinal adverse events specified in system organ class. Data were synthesised using NMA and a Bayesian framework. Results: In total, 27 studies were included in the analyses. Orally administered semaglutide $14 \mathrm{mg}$ QD was associated with significantly greater reductions in $\mathrm{HbA}_{1 \mathrm{c}}$ vs most comparators, and numerically greater reductions vs semaglutide $0.5 \mathrm{mg}$ once-weekly (QW), dulaglutide $1.5 \mathrm{mg}$ QW and liraglutide $1.8 \mathrm{mg}$ QD. $\mathrm{HbA}_{1 \mathrm{c}}$ reductions with semaglutide $1 \mathrm{mg}$ QW were numerically greater than those with orally administered semaglutide $14 \mathrm{mg}$ QD. Reductions in body weight for orally administered semaglutide $14 \mathrm{mg}$ QD were significantly greater than all comparators except semaglutide QW (both doses). Orally administered semaglutide QD $14 \mathrm{mg}$ was associated with statistically similar odds of experiencing gastrointestinal adverse events vs injectable GLP-1 RAs. Conclusion: Orally administered semaglutide $14 \mathrm{mg}$ QD as an add-on to 1-2 OADs is one of the most efficacious GLP-1 RAs for reducing 
$\mathrm{HbA}_{1 \mathrm{c}}$ and body weight at $26 \pm 4$ weeks. Orally administered semaglutide $14 \mathrm{mg}$ QD is well tolerated, with a safety profile in line with the GLP-1 RA class.

Funding: Novo Nordisk.

Keywords: Body weight; GLP-1 receptor agonist; Glycaemic control; $\mathrm{HbA}_{1 \mathrm{c}}$; Network meta-analysis; Orally administered semaglutide; Systematic literature review; Type 2 diabetes

\section{INTRODUCTION}

Type 2 diabetes (T2D) is a chronic and progressive metabolic disorder characterised by elevated levels of blood glucose (hyperglycaemia) [1]. Prolonged, suboptimal glycaemic control is associated with an increased risk of macrovascular (e.g. myocardial infarction, stroke and heart failure) and microvascular (e.g. retinopathy, neuropathy and nephropathy) complications which can reduce life expectancy, adversely impact patients' quality of life and increase the overall treatment costs associated with T2D [2-6].

The goal for the treatment of T2D is to prevent or delay complications and maintain quality of life, which requires good glycaemic control and management of cardiovascular (CV) risk factors $[7,8]$. Given the progressive nature of the disease, T2D often requires an intensification of treatment over time to achieve glycaemic targets, assessed by measuring glycated haemoglobin $\left(\mathrm{HbA}_{1 \mathrm{c}}\right)$ [7, 8]. Current treatment guidelines recommend that patients achieve and maintain a target $\mathrm{HbA}_{1 \mathrm{c}}$ level of either $<7 \% \quad(53 \mathrm{mmol} / \mathrm{mol}) \quad$ or $\quad$ of $\leq 6.5 \%$ ( $48 \mathrm{mmol} / \mathrm{mol})$ while minimising the risk of hypoglycaemia [7-11]. Despite clinical guideline recommendations, up to $50 \%$ of patients across Europe and the USA do not meet glycaemic targets $[12,13]$. This is due to several factors including poor adherence to treatment, delay or failure to initiate or intensify therapy (i.e. therapeutic inertia), as well as the suboptimal efficacy and side effects of some treatments $[14,15]$. Body weight control is also an important element of glycaemic management strategies, and it is estimated that approximately $90 \%$ of adults are overweight or obese at the time of T2D diagnosis [16]. Increased body weight is associated with an increased risk of CV disease, all-cause mortality and reduced quality of life among people with T2D [17-19]. Blood pressure, a surrogate marker of $\mathrm{CV}$ risk outcomes, is another outcome of interest in the management of T2D. Accordingly, clinical guidelines recommend adequate blood pressure control to reduce CV risk [20-22].

Targeting the incretin system has become an important therapeutic approach for treating T2D [23]. Glucagon-like peptide 1 receptor agonists (GLP-1 RAs) are incretin mimetics with proven benefits in terms of improving glycaemic control without increasing the risk of hypoglycaemia [24]. GLP-1 RAs provide significant body weight reduction, and $\mathrm{CV}$ risk reduction has also been observed with some GLP-1 RAs in patients with T2D with CV risk [24-28]. The American Diabetes Association and the European Association for the Study of Diabetes recommend GLP-1 RAs as either secondor third-line agents [i.e. as an add-on therapy to 1 or 2 oral antidiabetic drugs (OADs)] in the treatment algorithm [7]. Furthermore, GLP-1 RAs are specifically recommended for patients with T2D who are overweight or obese by the American Association of Clinical Endocrinologists, the Canadian Diabetes Association and UK guidelines [21, 29, 30].

Orally administered semaglutide is the first and only once-daily (QD) oral GLP-1 RA for the treatment of T2D. It has been extensively studied in the Peptide InnOvatioN for Early diabEtes tReatment (PIONEER) Phase III clinical trial programme [31-34]. The efficacy and safety of orally administered semaglutide QD in patients inadequately controlled on 1-2 OADs has been studied in PIONEER 2 [31], PIONEER 3 [32], PIONEER 4 [34] and PIONEER 7 [33]. PIONEER 4 provides evidence for orally administered semaglutide $14 \mathrm{mg}$ vs a GLP-1 RA in patients inadequately controlled on 1-2 OADs, showing significantly greater reductions in $\mathrm{HbA}_{1 \mathrm{c}}$ and body weight with orally administered semaglutide $14 \mathrm{mg}$ compared with liraglutide and placebo (both treatments + metformin \pm sodium/ glucose cotransporter 2 inhibitor, SGLT2i) [34]. 
The PIONEER programme also established that orally administered semaglutide QD is well tolerated [31-34] and that its safety profile is similar to that of liraglutide [34].

A wide range of treatment options are currently available to patients with T2D. Thus, understanding the relative clinical benefits of each treatment is very important to allow recommendations on their use within a limited budget. While PIONEER 4 provides robust evidence on the efficacy and safety of orally administered semaglutide QD vs liraglutide [34], evidence from head-to-head trials between orally administered semaglutide and other GLP1 RAs is limited. Hence, the objective of this study was to conduct a systematic literature review (SLR) and network meta-analysis (NMA) to assess the relative efficacy and safety of orally administered semaglutide $14 \mathrm{mg}$ QD compared with injectable GLP-1 RAs in patients with T2D inadequately controlled on 1-2 OADs.

\section{METHODS}

\section{Systematic Literature Review}

An SLR was performed in accordance with Preferred Reporting Items for Systematic Reviews and Meta-Analyses (PRISMA) guidelines [35] to identify randomised controlled trial (RCT) evidence on a wide range of T2D interventions (i.e. all currently used T2D pharmacotherapies) and patient populations. Methodology and results presented herein are specific to studies reporting on the efficacy and safety of orally administered semaglutide and injectable GLP-1 RAs in patients with T2D who are inadequately controlled on 1-2 OADs. Searches of MEDLINE ${ }^{\circledR}$, Embase and the Cochrane Library were initially performed via Ovid on 5 April 2016 and most recently updated on 2 January 2019 (see Table S1 in supplementary information for further details). Searches of conference proceedings were also carried out for the European Association for the Study of Diabetes, the International Society for Pharmacoeconomics and Outcomes Research (ISPOR), the International Diabetes Federation and the American Diabetes Association Scientific Sessions.
Following a study screening hierarchy for exclusion, all titles and abstracts identified through the literature searches were screened by two reviewers to assess whether they met the PICOS (population, interventions, comparators, outcomes, study design) selection criteria (supplementary information, Table S2). Once title and abstract screening were completed, any existing discrepancy between the reviewers regarding study selection was reconciled. The same two reviewers independently screened full-text articles for all studies included during the title and abstract screening phase. When a consensus between the two reviewers could not be reached, a senior reviewer provided arbitration. In addition, data from digital curves were extracted using digital extraction tools. Any discrepancies observed between the data extracted by the two analysts were adjudicated by a third analyst. This article is based on previously conducted studies and does not contain any studies with human participants or animals performed by any of the authors.

\section{NMA Methodology}

An NMA was performed in accordance with guidance from the National Institute for Health and Care Excellence (NICE), ISPOR and the Cochrane Institute [36-40], to assess the relative efficacy of orally administered semaglutide compared with GLP-1 RAs for the treatment of T2D as an add-on to 1-2 OADs. In the analysis, the primary intervention of interest was orally administered semaglutide $14 \mathrm{mg}$ QD and the primary comparators of interest were all licensed doses of injectable GLP-1 RAs-liraglutide, dulaglutide, exenatide twice-daily (BID), exenatide extended release, lixisenatide and subcutaneously administered semaglutide once-weekly (QW). Albiglutide was withdrawn from the market in 2018 [41] and therefore was not considered a relevant comparator in the NMA. GLP-1 RAs were often taken with other background antidiabetic medications in the trials. To reduce variability between populations across the different trials, the definition of the population receiving an add-on to 1-2 OADs was aligned as closely as possible to populations 
in the relevant PIONEER trials of orally administered semaglutide (the primary intervention of interest). The trial population in PIONEER 2 was patients inadequately controlled on metformin, and the trial populations in PIONEER 3, 4 and 7 were patients inadequately controlled on 1-2 OADs (metformin \pm sulfonylureas) in PIONEER 3 , metformin \pm SGLT2i in PIONEER 4 and 1-2 OADs (metformin, sulfonylureas, SGLT2i or thiazolidinediones) in PIONEER 7.

Trials assessing a patient population that aligned with PIONEER trials 2, 3, 4 or 7 were considered for analysis and consequently, trials which included only patients inadequately controlled on two OADs were excluded. Similarly, studies which included less than $90 \%$ of patients inadequately controlled on metformin monotherapy, or on one OAD that was not metformin, were excluded from the analysis to reflect standard of care and align with international guidelines [7].

The PIONEER programme used two different estimands. The treatment policy estimand evaluated the treatment effect for all randomised patients regardless of trial product discontinuation and use of rescue medication (data analysed using multiple imputation), whereas the trial product estimand evaluated the treatment effect for all randomised patients under the assumption that all patients remained on trial product for the entire planned duration of the trial and did not use rescue medication (data analysed using a mixed model for repeated measures) [42]. To allow for accurate comparisons with trials reporting data without the use of rescue medication, the trial product estimand from the PIONEER trials was used for this NMA.

The identified studies were assessed for data on at least one outcome of interest, as well as their potential to form a connected network. A feasibility analysis for generating an evidence network for the 20 outcomes of interest was also conducted (supplementary information, Table S2). The NMA was considered feasible for the following efficacy outcomes: change from baseline in $\mathrm{HbA}_{1 \mathrm{c}}$; proportion of patients achieving $\quad \mathrm{HbA}_{1 \mathrm{c}}<7 \% \quad(53 \mathrm{mmol} / \mathrm{mol})$ or $\leq 6.5 \%$ (48 $\mathrm{mmol} / \mathrm{mol})$; changes from baseline in body weight and blood pressure [i.e. systolic blood pressure (SBP) and diastolic blood pressure (DBP)], and safety outcomes including the proportion of patients experiencing any gastrointestinal (GI) adverse events (AEs) as specified in system organ class.

A normal likelihood, identity link model was used to perform all analyses of continuous outcomes. Where necessary, a shared parameter model was implemented to account for armlevel as well as trial-level data reported in the studies. A binomial likelihood (assuming a normal distribution) logit link model was used for the analysis of dichotomous outcomes. Both fixed effects and random effects models were run for each outcome, and the most suitable model was chosen on the basis of two criteria: the deviance information and the average posterior residual deviance.

The NMA models were implemented using WinBUGS software (MRC Biostatistics Unit, Cambridge, UK) [43] and employed a Bayesian framework with the use of uninformative prior distributions. Three Markov Monte Carlo chains were used, starting from different initial values of selected unknown parameters with a burn-in of 50,000 iterations. Convergence for all models was assessed by analysing history and density plots, and Brooks-Gelman-Rubin diagnostic plots [44]. In addition, autocorrelation plots were assessed to detect the presence of autocorrelation in the chains. Following this, model convergence inferences were made from data obtained by sampling for a further 10,000 iterations on the three chains.

Median treatment differences or odds ratios (ORs) and an associated 95\% credible interval (CrI) are presented for the NMA results. For the continuous outcomes $\mathrm{HbA}_{1 \mathrm{c}}(\%)$, body weight $(\mathrm{kg})$, SBP and DBP (mmHg), a treatment associated with a greater mean reduction from baseline is favoured. For efficacy dichotomous outcomes, a treatment associated with an increase in the OR (e.g. higher odds for achieving a $\mathrm{HbA}_{1 \mathrm{c}}$ level $<7 \%$ ) is favoured. For GI AEs, a treatment associated with a decrease in the OR is favoured.

In Bayesian statistics, it is considered that differences exist only where the CrI does not include 0.0 for treatment differences, or 1.0 for ORs. In some cases, orally administered 
semaglutide may be associated with a numerical reduction/increase against a comparator; however, it is assumed that there is no difference between treatments unless the CrI excludes 0.0 (for treatment differences), or 1.0 (for ORs).

The median ranks of each treatment are also provided in the supplementary information (Table S14). A treatment with a median rank of 1 is considered the best. If two drugs are both ranked as the second highest, they will both be given a lower median rank score (i.e. score 3). The surface under the cumulative ranking curve (SUCRA) is also presented in the supplementary information (Table S13). SUCRA values vary between $0 \%$ and 100\%; a higher SUCRA value indicates an increased likelihood that a treatment is in the top rank or one of the top ranks [45]. This single numeric value can be a helpful simplification of information about the effect of each treatment, enabling easier interpretation of the many alternative results that are often calculated within an NMA network.

NMAs estimate treatment effects by combining evidence from clinical trials. This involves combining direct and indirect measures of effect, the findings of which may not always be aligned with each other. Therefore, it is important to examine consistency between the two 'sources' of evidence. Hence, where treatment loops were present in the network diagrams, these were statistically evaluated for inconsistency using Bucher's method [39]. Additional informal checks were also performed by comparing the direct study data with the results of the NMA.

This article does not contain any new studies with human or animal subjects performed by any of the authors.

\section{RESULTS}

\section{Identified Publications}

In total, 108 publications reporting on 71 unique RCTs were included in the SLR; an overall PRISMA flow diagram of the SLR (searches performed between 5 April 2016 and 2 January 2019) is presented in Fig. 1. Individual PRISMA flow diagrams (for each search update between 2016 and 2019) and a list of included trials are provided in the supplementary information (Fig. S1 and Table S3, respectively). Of the 71 trials, 47 trials were considered as potentially relevant for inclusion in the NMA. The process of excluding the remaining 24 studies is detailed in Fig. S2. All 47 trials considered in the NMA formed a connected network, which allowed for the comparison of orally administered semaglutide QD (14 mg dose) with dulaglutide QW (0.75 mg and $1.5 \mathrm{mg}$ doses), liraglutide QD (1.2 mg and $1.8 \mathrm{mg}$ doses) exenatide BID $(5.0 \mu \mathrm{g}$ and $10.0 \mu \mathrm{g}$ doses), exenatide extended release QW (2 mg dose), semaglutide QW (0.5 $\mathrm{mg}$ and $1 \mathrm{mg}$ doses) and lixisenatide QD (20 $\mu \mathrm{g}$ dose). All secondary comparators were removed from the network as they did not contribute to any connections in the network, except for placebo, sitagliptin and insulin glargine which were secondary comparators that connected primary comparators of interest. Therefore, four individual treatment arms (i.e. the pioglitazone arm of the DURATION-2 trial, the orally administered semaglutide $3 \mathrm{mg}$ and $7 \mathrm{mg}$ arms of the PIONEER 3 trial and fixed combination of insulin glargine and lixisenatide arm of LixiLan-O) and seven complete trials [31, 46-51] were removed from the networks. Furthermore, PIONEER 7 was removed because of its different trial design (assessing flexible dosing for orally administered semaglutide). This resulted in a total of 39 trials remaining in the network [32, 34, 46, 52-87].

In this network, multiple studies informed the treatment effect for both insulin glargine and lixisenatide. Pooling the insulin glargine arms into a single treatment node was considered clinically appropriate. Similarly, for the lixisenatide treatment arms, though differences were observed in the titration strategies, these were considered clinically similar such that they could be pooled into a single lixisenatide $20 \mu \mathrm{g}$ QD arm. The only exception to pooling lixisenatide $20 \mu \mathrm{g}$ doses was with the morning and evening administration reported in the GET GOAL-M trial, as its primary objective was specifically to investigate morning vs evening administration of this treatment dose.

The 39 trials retained in the network were examined for time points at which data were 


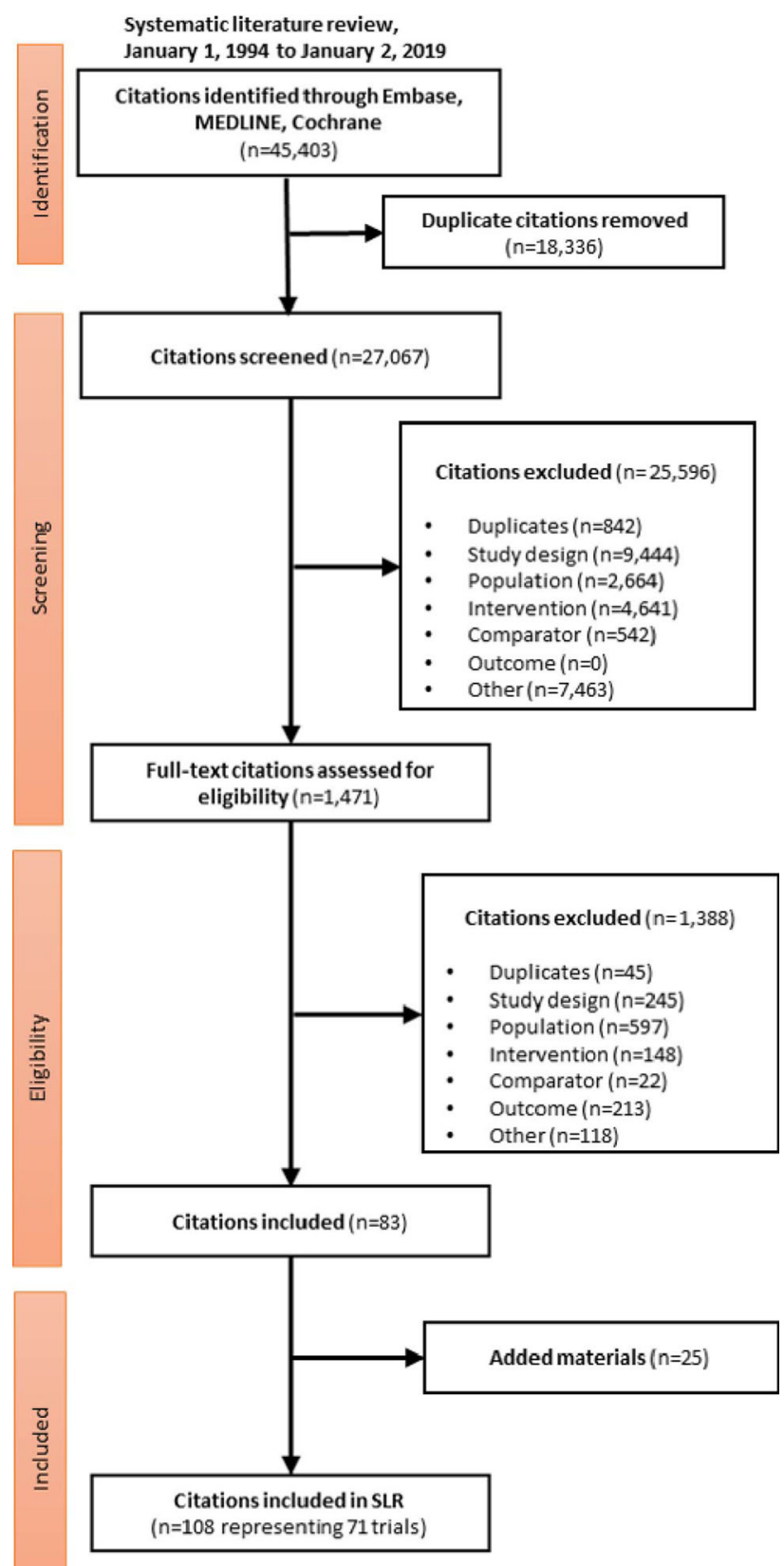

Fig. 1 PRISMA flow diagram of the SLR (systematic literature review)

available for at least one outcome (supplementary information, Table S4). All 39 trials reported data for at least one outcome of interest between 23 and 28 weeks. The level of response to treatment within 4 weeks of the target week was assumed unlikely to vary considerably; therefore, it was considered clinically relevant to analyse each outcome at $26 \pm 4$ weeks (approximately 6 months). The study design and patient characteristics for the 39 trials are presented in the supplementary information (Table S5). Overall, the risk of bias was low across the 39 studies, with the greatest bias risk being related to elements of unclear or lack of study blinding and discontinuations. 


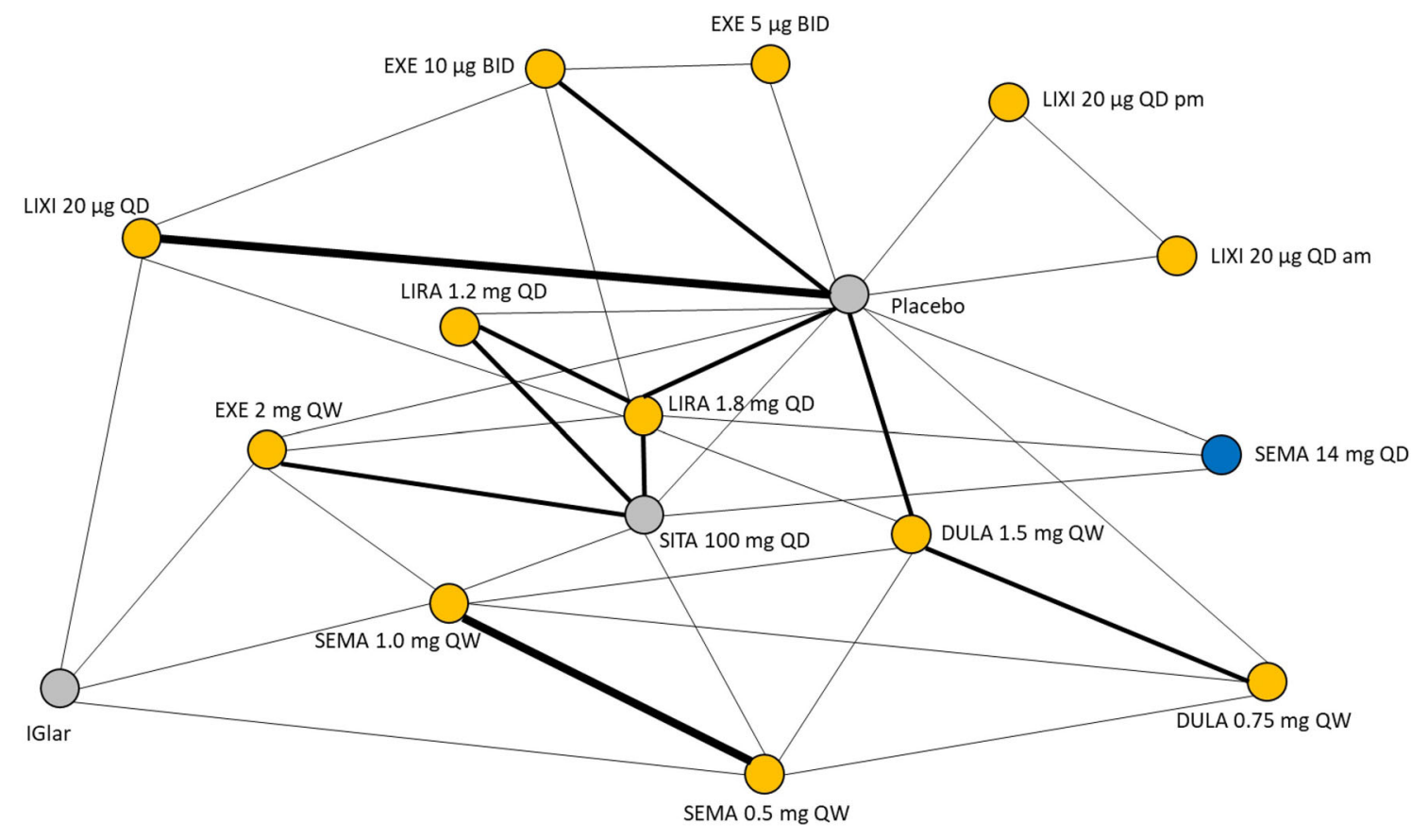

Fig. 2 Base case evidence network. Line thickness corresponds to the number of trials contributing to the comparison between two interventions-the thickest equates to three trials, while the thinnest equates to one trial. The blue node indicates the primary intervention of interest, orange nodes indicate a primary comparator of

The majority of trials were considered sufficiently homogenous to be combined in the analysis. However, eight studies identified in the SLR were considered as potential sources of heterogeneity due to study design and patient characteristics. Of these eight studies, Derosa 2012 [62] enrolled treatment-naive patients who received metformin for 8 months prior to treatment randomisation; Van Gaal 2014 [68] was a study in young (mean age of 43 years) and obese (mean body mass index of $36.8 \mathrm{~kg} / \mathrm{m}^{2}$ ) patients; and six studies (Araki 2015 [70], GET GOAL-M Asia [85], Inagaki 2012 [79], Ji 2013 [76], AWARD-CHN2 [81] and Zang 2016 [69]) enrolled only Asian patients. Furthermore, four trials were excluded from the analyses because of heterogeneity in the background treatment and interventions assessed. DURATION-NEO-1 [82] included patients with $0-3$ previous OADs, and relevant subgroup data for the population of interest for inclusion in the base case NMA were not available. HARMONY-1 [74], interest, and grey nodes indicate a secondary comparator. am morning, BID twice-daily, DULA dulaglutide, EXE exenatide, IGlar insulin glargine, LIRA liraglutide, LIXI lixisenatide, $p m$ evening, $Q D$ once-daily, $Q W$ once-weekly, SEMA semaglutide, SITA sitagliptin

HARMONY-3 [46] and HARMONY-4 [75] assessed albiglutide which was withdrawn from the market in 2018 [41]. It was therefore decided to exclude these 12 studies from the analysis, yielding a total of 27 studies for inclusion in the base case evidence network [32, 34, 52-61, 63$67,71-73,77,78,80,83,84,86,87]$.

\section{NMA Results}

Overall, 27 RCTs reporting outcomes of interest at $26 \pm 4$ weeks follow-up were included in the analyses; the evidence network is shown in Fig. 2. In the analysis for the change from baseline in $\mathrm{HbA}_{1 \mathrm{c}}$ and body weight, proportion of patients achieving $\mathrm{HbA}_{1 \mathrm{c}}$ level of $<7 \%$ or $\leq 6.5 \%$, and GI AEs, the random effects model was chosen as it provided a better fit in terms of deviance information criterion compared with the fixed effects model. In contrast, for the analyses assessing the change from baseline in 
a
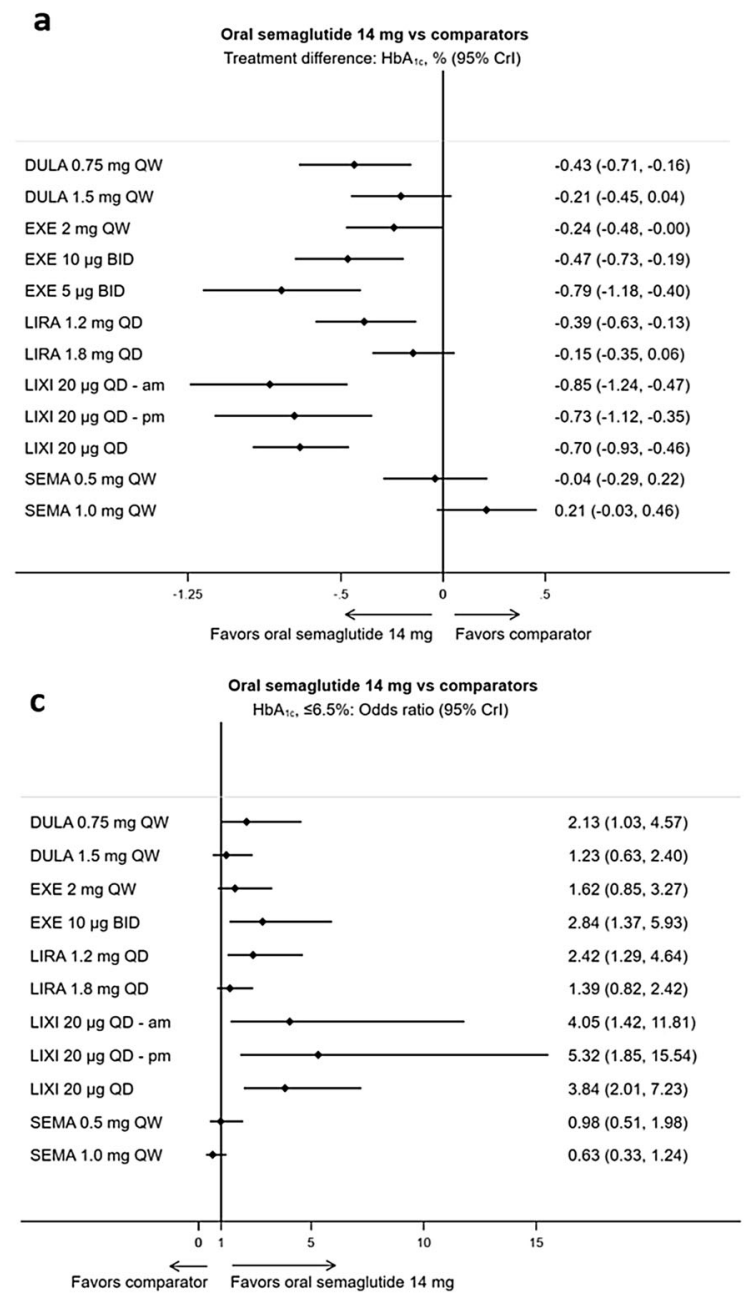

Fig. 3 Forest plots of the NMA results: efficacy outcomes for orally administered semaglutide $14 \mathrm{mg}$ QD vs comparators. Treatment differences are considered significant when the 95\% CrI excludes 0.0. Odds ratios are considered significant when the 95\% CrI excludes 1.0. The NMA results are presented as forest plots for a change from baseline in $\mathrm{HbA}_{1 \mathrm{c}}$, b proportion of patients achieving a

SBP and DBP, the fixed effects model was chosen as it provided a better fit than the random effects model. The results of the NMA are presented as treatment differences or ORs (orally administered semaglutide QD vs comparator) in Fig. 3. The full matrices of relative treatment effect results are presented in the supplementary information (Tables S6-S12). The associated treatment ranks (SUCRA and median rank) for each outcome are also presented in the supplementary information (Tables S13 and b

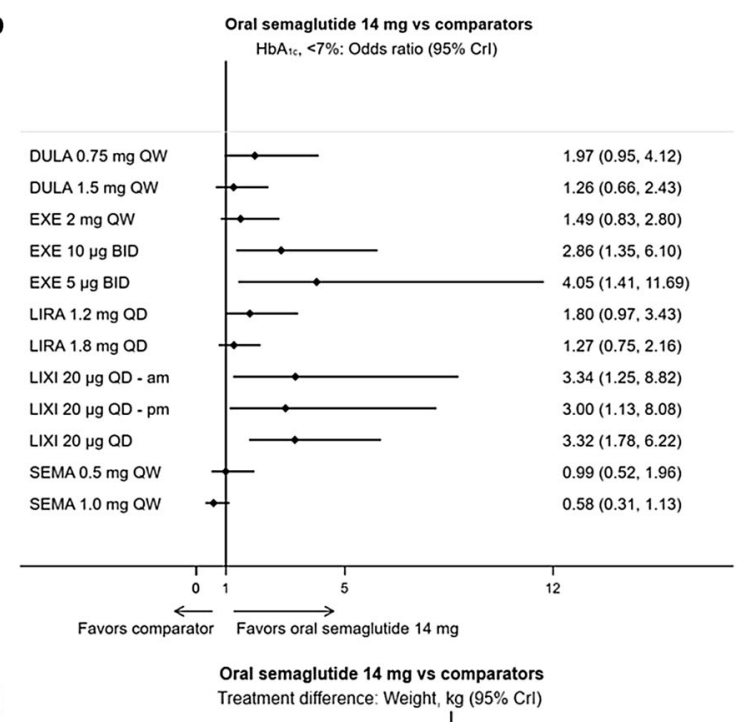

d

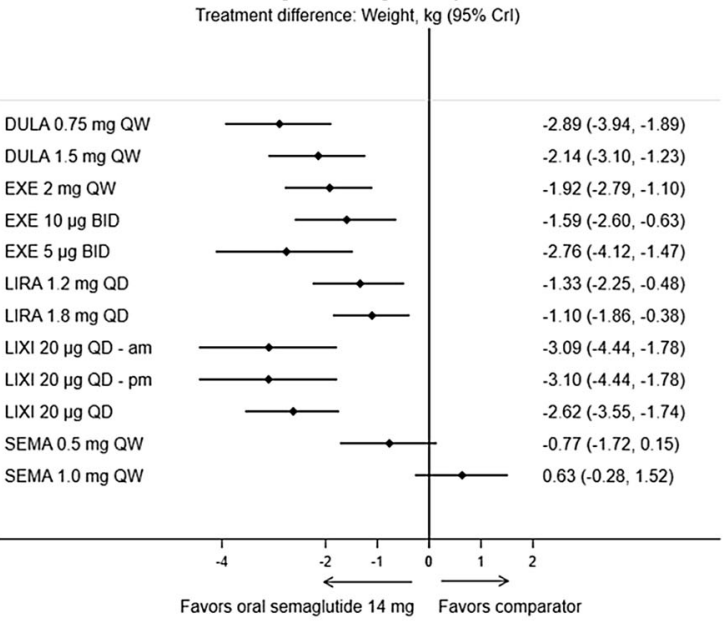

$\mathrm{HbA}_{1 \mathrm{c}}$ level $<7 \%$ or $\mathbf{c} \mathrm{HbA}_{1 \mathrm{c}}$ level $\leq 6.5 \%$, $\mathbf{d}$ change from baseline in body weight. am morning, BID twicedaily, $C r I$ credible interval, DULA dulaglutide, EXE exenatide, $H b A_{1 c}$ glycated haemoglobin, LIRA liraglutide, LIXI lixisenatide, NMA network meta-analysis, $p m$ evening, $Q D$ once-daily, $Q W$ once-weekly, SEMA semaglutide

S14, respectively). In addition, the estimated absolute treatment effects for each outcome are available in supplementary information (Table S15).

\section{Glycaemic Control}

All 27 RCTs reported data on the change from baseline in $\mathrm{HbA}_{1 \mathrm{c}}$ (supplementary information, Table S16); the evidence network for this 
analysis is shown in Fig. 2. The results (Fig. 3a; Table S6) showed that orally administered semaglutide $14 \mathrm{mg}$ QD was associated with a significantly greater reduction in $\mathrm{HbA}_{1 \mathrm{c}}$ vs dulaglutide $0.75 \mathrm{mg}$ QW, exenatide BID (both doses), exenatide $2 \mathrm{mg}$ QW, liraglutide $1.2 \mathrm{mg}$ QD and lixisenatide QD (all doses). Reductions in $\mathrm{HbA}_{1 \mathrm{c}}$ for orally administered semaglutide $14 \mathrm{mg}$ QD were numerically greater, although not statistically significant, compared with semaglutide $0.5 \mathrm{mg}$ QW, dulaglutide $1.5 \mathrm{mg}$ QW and liraglutide $1.8 \mathrm{mg}$ QD. Conversely, semaglutide $1 \mathrm{mg}$ QW was associated with numerically greater $\mathrm{HbA}_{1 \mathrm{c}}$ reductions vs orally administered semaglutide $14 \mathrm{mg}$ QD. No comparators were identified as significantly better than orally administered semaglutide $14 \mathrm{mg}$ QD.

No significant inconsistency was detected in the network for the change from baseline in $\mathrm{HbA}_{1 \mathrm{c}}(p>0.05)$, except for one loop of evidence (insulin glargine; exenatide $2 \mathrm{mg} \mathrm{QW}$; semaglutide $1 \mathrm{mg}$ QW) for which significant inconsistency $(p=0.006)$ was detected. When the mean difference in change from baseline between semaglutide $1 \mathrm{mg}$ QW and exenatide $2 \mathrm{mg}$ QW was considered, the effect size direction was the same for both direct and indirect comparisons (direct: -0.36 ; 95\% confidence interval (CI) $-0.52,-0.20$; indirect: -0.74 ; $95 \% \mathrm{CI}-0.96,-0.52)$; consequently, the NMA also concurred with the direction of effect $(-0.45 ; 95 \%$ CrI $-0.64,-0.27)$. While the magnitude of effect differed between the direct, indirect and NMA results, the significant treatment difference favouring semaglutide $1 \mathrm{mg}$ QW over exenatide $2 \mathrm{mg}$ QW remained in each analysis. Exploratory analyses concluded that the impact on the results of the NMA due to inconsistency in this loop was minimal.

NMAs were also feasible for two other $\mathrm{HbA}_{1 \mathrm{c}}$ outcomes: $\mathrm{HbA}_{1 \mathrm{c}}$ level $<7 \%$ and $\mathrm{HbA}_{1 \mathrm{c}}$ level $\leq 6.5 \%$. The evidence networks and data supporting these analyses are shown in the supplementary information (Fig. S3, and Tables S17 and S18, respectively). In line with the results from the analysis of the change from baseline in $\mathrm{HbA}_{1 \mathrm{c}}$, orally administered semaglutide $14 \mathrm{mg}$ QD was associated with significantly higher odds of achieving a $\mathrm{HbA}_{1 \mathrm{c}}$ level $<7 \%$ vs exenatide BID (5 $\mu \mathrm{g}$ and $10 \mu \mathrm{g}$ doses) and all dosing schedules of lixisenatide $20 \mu \mathrm{g}$ (Fig. 3b; Table S7). Orally administered semaglutide $14 \mathrm{mg}$ QD was also associated with significantly higher odds of achieving a $\mathrm{HbA}_{1 \mathrm{c}}$ level $\leq 6.5 \%$ vs dulaglutide $0.75 \mathrm{mg} \mathrm{QW}$, exenatide $10 \mu \mathrm{g}$ BID, liraglutide $1.2 \mathrm{mg}$ QD and all dosing schedules of lixisenatide $20 \mu \mathrm{g}$ (Fig. 3c; Table S8). The NMA results also suggested that orally administered semaglutide $14 \mathrm{mg}$ QD was associated with higher odds of achieving target $\mathrm{HbA}_{1 \mathrm{c}}$ levels of $<7 \%$ and $\leq 6.5 \%$ vs all comparators, except semaglutide QW (both doses), although not all comparisons were statistically significant. These analyses indicate that the improved $\mathrm{HbA}_{1 \mathrm{c}}$ reduction observed with orally administered semaglutide $14 \mathrm{mg}$ QD vs injectable GLP-1 RAs increases the probability of achieving the recommended glycaemic targets.

No inconsistency was detected in most evidence loops in the networks for the proportion of patients achieving a $\mathrm{HbA}_{1 \mathrm{c}}$ level $<7 \%$ or $\leq 6.5 \%$. However, inconsistency was detected in one loop for $\mathrm{HbA}_{1 \mathrm{c}}$ level $<7 \%$ (placebo; sitagliptin $100 \mathrm{mg}$ QD; orally administered semaglutide $14 \mathrm{mg}$ QD), and one loop (placebo; liraglutide $1.8 \mathrm{mg}$ QD; lixisenatide $20 \mu \mathrm{g}$ QD) for both $\mathrm{HbA}_{1 \mathrm{c}}$ outcomes. As with the change from baseline $\mathrm{HbA}_{1 \mathrm{c}}$ analysis, exploratory analyses concluded that there was limited impact on the results of the NMA due to the inconsistency in these loops. Across these analyses, orally administered semaglutide $14 \mathrm{mg}$ QD was one of the highest ranked GLP-1 RAs, achieving SUCRA values of $86-94 \%$ (Table S13) and median ranks of 2-3 (Table S14). Together, these treatment ranks indicated that orally administered semaglutide $14 \mathrm{mg}$ QD is the second most clinically efficacious treatment in terms of reduction of $\mathrm{HbA}_{1 \mathrm{c}}$, and the third most effective for achieving a $\mathrm{HbA}_{1 \mathrm{c}}$ level $<7 \%$ or $\leq 6.5 \%$ at $26 \pm 4$ weeks, with semaglutide QW being the only treatment ranked higher.

\section{Body Weight}

In total, 27 trials reported the change from baseline in body weight (supplementary 
information, Table S19) and the evidence network is shown in Fig. S3. The results showed that orally administered semaglutide $14 \mathrm{mg}$ QD was associated with a significantly greater reduction in body weight vs all GLP-1 RA comparators except semaglutide QW $(0.5 \mathrm{mg}$ and $1 \mathrm{mg}$ doses) (Fig. 3d; Table S9). No significant inconsistency was detected in this network $(p>0.05)$. Together, the SUCRA values and median ranks indicate that orally administered semaglutide $14 \mathrm{mg}$ QD is the second most efficacious GLP-1 RA in terms of body weight reduction at $26 \pm 4$ weeks (Table S13 and S14).

\section{Blood Pressure}

Blood pressure was assessed in terms of changes from baseline in SBP and DBP. In total, 21 trials reported the change from baseline in SBP, and 20 trials reported the change from baseline in DBP (supplementary information, Tables S20 and S21, respectively). The evidence network for both outcomes is shown in Fig. S3. The analysis suggested that orally administered semaglutide $14 \mathrm{mg}$ QD was associated with a similar reduction in SBP vs all GLP-1 RA comparators, except semaglutide $1 \mathrm{mg}$ QW (supplementary information, Table S10). Orally administered semaglutide $14 \mathrm{mg}$ QD was also associated with a similar reduction in DBP vs all GLP-1 RA comparators (supplementary information, Table S11). No significant inconsistency was detected in the loops of evidence for both networks. The ranking and SUCRA values for blood pressure outcomes are reported in supplementary information (Tables S13 and S14, respectively).

\section{Safety Outcomes}

One of the most commonly cited reasons for treatment discontinuation with GLP-1 RAs is GI AEs $[88,89]$. Therefore, an analysis of the incidence of the most commonly reported GI AEs (e.g. nausea, vomiting, diarrhoea) was performed in this NMA. In total, 17 trials reported the proportion of patients experiencing GI AEs (supplementary information, Table S22) and the evidence network is shown in Fig. S3. The analysis indicated that orally administered semaglutide $14 \mathrm{mg}$ QD was associated with statistically similar odds of experiencing GI AEs compared with injectable GLP-1 RAs (supplementary information, Table S12). No significant inconsistency was detected in the evidence network for GI AEs $(p>0.05)$, except in one loop of evidence for which the direction of the effect was the same and there was some overlap of $95 \%$ CrIs.

\section{DISCUSSION}

The objective of this study was to assess the relative efficacy and safety of orally administered semaglutide $14 \mathrm{mg}$ QD compared with injectable GLP-1 RAs in patients with T2D inadequately controlled on 1-2 OADs. The analyses showed that orally administered semaglutide $14 \mathrm{mg}$ QD was associated with a significantly greater reduction in $\mathrm{HbA}_{1 \mathrm{c}}$ at $26 \pm 4$ weeks vs most GLP-1 RA comparators, and with numerically greater reductions vs semaglutide $0.5 \mathrm{mg}$ QW, dulaglutide $1.5 \mathrm{mg}$ QW and liraglutide $1.8 \mathrm{mg}$ QD. Conversely, semaglutide $1 \mathrm{mg}$ QW was associated with numerically greater $\mathrm{HbA}_{1 \mathrm{c}}$ reductions compared with orally administered semaglutide $14 \mathrm{mg}$ QD. Once-daily orally administered semaglutide also provided a significantly greater reduction in body weight vs all GLP-1 RA comparators except semaglutide QW $(0.5 \mathrm{mg}$ and $1 \mathrm{mg}$ doses) and was associated with similar reductions in SBP and DBP vs all GLP-1 RA comparators, except semaglutide $1 \mathrm{mg}$ QW. Results also suggest that the improved $\mathrm{HbA}_{1 \mathrm{c}}$ reduction observed with orally administered semaglutide $14 \mathrm{mg}$ QD vs injectable GLP-1 RAs, except semaglutide QW (both doses), increases the probability of achieving the recommended glycaemic target levels of $<7 \%$ or $\leq 6.5 \%$, although not all comparisons were statistically significant. The findings from these analyses are supported by treatment ranks and SUCRA values which together indicate that orally administered semaglutide $14 \mathrm{mg}$ QD is the second best treatment, after semaglutide $1 \mathrm{mg} \mathrm{QW}$, for reducing $\mathrm{HbA}_{1 \mathrm{c}}$ levels and body weight at $26 \pm 4$ weeks. 
A good balance between improvements in efficacy and the risk of AEs is important for effective treatment. Gastrointestinal AEs are commonly cited reasons for discontinuing treatment with GLP-1 RAs $[88,89]$. Our analysis found that orally administered semaglutide $14 \mathrm{mg}$ was associated with similar odds of experiencing GI AEs, including nausea, vomiting and diarrhoea, when compared with injectable GLP-1 RAs. This is in line with the findings from the PIONEER clinical trial programme which demonstrated that orally administered semaglutide is well tolerated [31-34] and has a safety profile similar to that of liraglutide [34]. Inconsistency was detected in one loop of the evidence network for GI AEs. Along with the subjectivity associated with reporting of GI AEs that may impact the relative treatment effect, potential effect modifiers for outcomes were identified including diet, eating patterns and use of medication to ameliorate GI issues. However, as these parameters were not widely reported across studies in the network, a meta-regression to examine the impact of these factors on the outcome was not feasible. Furthermore, this loop consisted of only a single trial to inform each treatment pair and it was not possible to identify which trial was "deviant" amongst these, thus causing the inconsistency.

To our knowledge, this is the first study assessing the relative efficacy and safety of orally administered semaglutide $14 \mathrm{mg}$ as an add-on therapy to 1-2 OADs compared with currently available GLP-1 RAs. Four recent NMAs assessed the relative efficacy and safety of GLP-1 RAs (not including orally administered semaglutide $14 \mathrm{mg}$ ) [90-93] of which one considered patients with T2D inadequately controlled on 1-2 OADs as its target population [93]. The findings from our NMA in terms of GLP-1 RAs' relative efficacy and ranking are consistent with those reported in the NMA by Witkowski et al. [93].

Findings from this NMA are robust on the basis of the number and homogeneity of trials included in the evidence network, as well as the alignment of its methodology with clinical practice guidance from NICE, ISPOR and the Cochrane Institute [36-40]. Furthermore, all the trials included in the NMA were identified in an SLR, ensuring that all available evidence was captured. The network also considered RCTs assessing GLP-1 RAs as an add-on to 1-2 OADs to include all the relevant PIONEER trials that assessed orally administered semaglutide as an add-on to OADs. Importantly, the NMA focused on patients with T2D previously receiving 1-2 OADs who are a relevant segment of patients routinely receiving GLP-RAs.

The NMA was subject to common limitations, including some heterogeneity in populations across the different trials, the time points reported across the trials, publication bias in the studies included in the SLR, and inconsistency in some evidence networks. To minimise variability between trial populations, the definition of population receiving an addon to 1-2 OADs was closely aligned to populations in the relevant trials of orally administered semaglutide; therefore, only trials assessing a patient population that aligned with PIONEER trials 2, 3, 4 or 7 were considered in the analysis. The heterogeneity in the reported time points was addressed by using a common and well-accepted approach of time point window [91, 92, 94]. Overall, the risk of bias was considered low across the studies. While the majority of trials in the analyses were blinded, 20 trials were open label, which could potentially increase the risk of performance bias [95]. Inconsistency was also detected in some of the networks in the analyses at $26 \pm 4$ weeks. However, removing evidence to reduce inconsistency is not always considered best practice [39]. The NMA estimates are a compromise between direct and indirect evidence and therefore removing direct evidence for one treatment comparison (which is also used as indirect evidence in other treatment comparisons) means that the NMA results are likely to vary and it would be impossible to identify which indirect evidence is not consistent with the direct evidence. Therefore, the presence of inconsistency is a limitation of the NMA results for outcomes where inconsistency was detected. 


\section{CONCLUSION}

Orally administered semaglutide $14 \mathrm{mg}$ QD as an add-on to 1-2 OADs is one of the most efficacious GLP-1 RAs for reducing $\mathrm{HbA}_{1 \mathrm{c}}$ levels and body weight, and for achieving target $\mathrm{HbA}_{1 \mathrm{c}}$ levels at $26 \pm 4$ weeks. In addition, the similar odds of experiencing GI AEs in comparison with other GLP-1 RAs indicate that orally administered semaglutide is well tolerated and not associated with additional burden to patients.

\section{ACKNOWLEDGEMENTS}

Funding. Sponsorship for this study and the Rapid Service Fee were funded by Novo Nordisk.

Medical Writing, Editorial and Other Assistance. Medical writing and editorial assistance in the preparation of this manuscript was provided by Sonia Alesso of DRG Abacus; support for this assistance was funded by Novo Nordisk. The SLR was conducted by DRG Abacus. For the NMA, data analysis was performed Abby Paine of Zedediah Consulting and feasibility assessment was conducted by Jatin Gupta of DRG Abacus. SLR and NMA were both funded by Novo Nordisk. The authors would like to thank Srikanth Deenadayalan for reviewing the manuscript.

Authorship. All named authors meet the International Committee of Medical Journal Editors (ICMJE) criteria for authorship for this manuscript, take responsibility for the integrity of the work, and have given final approval to the version to be published.

Disclosures. Solomon Nuhoho is an employee of Novo Nordisk. Brian Bekker Hansen is an employee and shareholder of Novo Nordisk. Tam Dang-Tan is an employee and shareholder of Novo Nordisk. Jatin Gupta is an employee of DRG Abacus. Mary Fletcher-Louis is an employee of DRG Abacus. Abby Paine is the owner of Zedediah Consulting.
Compliance with Ethics Guidelines. This article is based on previously conducted studies and does not contain any studies with human participants or animals performed by any of the authors.

Data Availability. All data generated or analysed during this study are included in this published article as supplementary information files.

Open Access. This article is distributed under the terms of the Creative Commons Attribution-NonCommercial 4.0 International License (http://creativecommons.org/licenses/ by-nc/4.0/), which permits any noncommercial use, distribution, and reproduction in any medium, provided you give appropriate credit to the original author(s) and the source, provide a link to the Creative Commons license, and indicate if changes were made.

\section{REFERENCES}

1. Forbes JM, Cooper ME. Mechanisms of diabetic complications. Physiol Rev. 2013;93:137-88.

2. Caro JJ, Ward AJ, O'Brien JA. Lifetime costs of complications resulting from type 2 diabetes in the U.S. Diabetes Care. 2002;25:476-81.

3. Diabetes UK. Facts and stats 2016. https://www. diabetes.org.uk/Documents/Position\%20statemen ts/DiabetesUK_Facts_Stats_Oct16.pdf. Accessed 14 Aug 2019.

4. Hex N, Bartlett C, Wright D, Taylor M, Varley D. Estimating the current and future costs of type 1 and type 2 diabetes in the UK, including direct health costs and indirect societal and productivity costs. Diabet Med. 2012;29:855-62.

5. Stratton IM, Adler AI, Neil HA, et al. Association of glycaemia with macrovascular and microvascular complications of type 2 diabetes (UKPDS 35): prospective observational study. BMJ. 2000;321:405-12.

6. Williams R, Van Gaal L, Lucioni C. Assessing the impact of complications on the costs of type II diabetes. Diabetologia. 2002;45:S13-7.

7. Davies MJ, D'Alessio DA, Fradkin J, et al. Management of hyperglycemia in type 2 diabetes, 2018. A 
consensus report by the American Diabetes Association (ADA) and the European Association for the Study of Diabetes (EASD). Diabetes Care. 2018;2018(41):2669-701.

8. American Diabetes Association. Standards of medical care in diabetes. Diabetes Care. 2019;42:S61-70.

9. Handelsman Y, Bloomgarden ZT, Grunberger G, et al. American Association of Clinical Endocrinologists and American College of Endocrinologyclinical practice guidelines for developing a diabetes mellitus comprehensive care plan-2015-executive summary. Endocr Pract. 2015;21:413-37.

10. International Diabetes Federation (IDF). Clinical practice recommendations for managing type 2 diabetes in primary care 2017. https://www.idf.org/ e-library/guidelines/128-idf-clinical-practice-recom mendations-for-managing-type-2-diabetes-in-prim ary-care.html. Accessed 14 Aug 2019.

11. American Association of Clinical Endocrinologists and American College of Endocrinology. AACE/ ACE comprehensive type 2 diabetes management algorithm; 2019. https://www.aace.com/pdfs/ diabetes/AACE_2019_Diabetes_Algorithm_FINAL_ ES.pdf. Accessed 14 Aug 2019.

12. Carls G, Huynh J, Tuttle E, Yee J, Edelman SV. Achievement of glycated hemoglobin goals in the US remains unchanged through 2014. Diabetes Ther. 2017;8:863-73.

13. de Pablos-Velasco P, Parhofer KG, Bradley C, et al. Current level of glycaemic control and its associated factors in patients with type 2 diabetes across Europe: data from the PANORAMA study. Clin Endocrinol (Oxf). 2014;80:47-56.

14. Khan H, Lasker SS, Chowdhury TA. Exploring reasons for very poor glycaemic control in patients with type 2 diabetes. Prim Care Diabetes. 2011;5:251-5.

15. Reach G, Pechtner V, Gentilella R, Corcos A, Ceriello A. Clinical inertia and its impact on treatment intensification in people with type 2 diabetes mellitus. Diabetes Metab. 2017;43:501-11.

16. Carnethon MR, De Chavez PJ, Biggs ML, et al. Association of weight status with mortality in adults with incident diabetes. JAMA. 2012;308:581-90.

17. Bodegard J, Sundstrom J, Svennblad B, Ostgren CJ, Nilsson PM, Johansson G. Changes in body mass index following newly diagnosed type 2 diabetes and risk of cardiovascular mortality: a cohort study of 8486 primary-care patients. Diabetes Metab. 2013;39:306-13.
18. Coffey JT, Brandle M, Zhou H, et al. Valuing healthrelated quality of life in diabetes. Diabetes Care. 2002;25:2238-43.

19. Oldridge NB, Stump TE, Nothwehr FK, Clark DO. Prevalence and outcomes of comorbid metabolic and cardiovascular conditions in middle- and olderage adults. J Clin Epidemiol. 2001;54:928-34.

20. Authors/Task Force Members, Ryden L, Grant PJ, et al. ESC guidelines on diabetes, pre-diabetes, and cardiovascular diseases developed in collaboration with the EASD: The Task Force on diabetes, pre-diabetes, and cardiovascular diseases of the European Society of Cardiology (ESC) and developed in collaboration with the European Association for the Study of Diabetes (EASD). Eur Heart J. 2013;34:3035-87.

21. Garber AJ, Abrahamson MJ, Barzilay JI, et al. Consensus statement by the American Association of Clinical Endocrinologists and American College of Endocrinology on the comprehensive type 2 diabetes management algorithm-2019 Executive Summary. Endocr Pract. 2019;25:69-100.

22. Lorber D. Importance of cardiovascular disease risk management in patients with type 2 diabetes mellitus. Diabetes Metab Syndr Obes. 2014;7:169-83.

23. Garber AJ. Long-acting glucagon-like peptide 1 receptor agonists: a review of their efficacy and tolerability. Diabetes Care. 2011;34(Suppl 2):S279-84.

24. Prasad-Reddy L, Isaacs D. A clinical review of GLP-1 receptor agonists: efficacy and safety in diabetes and beyond. Drugs Context. 2015;4:212283.

25. Marso SP, Daniels GH, Brown-Frandsen K, et al. Liraglutide and cardiovascular outcomes in type 2 diabetes. N Engl J Med. 2016;375:311-22.

26. Marso SP, Bain SC, Consoli A, et al. Semaglutide and cardiovascular outcomes in patients with type 2 diabetes. N Engl J Med. 2016;375:1834-44.

27. Isaacs D, Prasad-Reddy L, Srivastava SB. Role of glucagon-like peptide 1 receptor agonists in management of obesity. Am J Health Syst Pharm. 2016;73:1493-507.

28. Gerstein HC, Colhoun HM, Dagenais GR, et al. Dulaglutide and cardiovascular outcomes in type 2 diabetes (REWIND): a double-blind, randomised placebo-controlled trial. Lancet. 2019;394:121-30.

29. Scottish Intercollegiate Guidelines Network. SIGN 154-a national clinical guideline: pharmacological management of glycaemic control in people with type 2 diabetes; 2017. http://www.sign.ac.uk/assets/ sign154.pdf. Accessed 14 Aug 2019. 
30. Canadian Diabetes Association Clinical Practice Guidelines Expert Committee. Pharmacologic management of type 2 diabetes: November 2016 interim update. Can J Diabetes. 2016;40:193-5.

31. Novo Nordisk. Data on file. PIONEER 2-oral semaglutide vs. SGLT-2 Inhibitor CTR, version 1.0 03 October 2018. A 52-week randomised, open-label, active-controlled trial to investigate the efficacy and safety of oral semaglutide versus empagliflozin in subjects with type 2 diabetes mellitus; 2018 .

32. Rosenstock J, Allison D, Birkenfeld AL, et al. Effect of additional oral semaglutide vs sitagliptin on glycated hemoglobin in adults with type 2 diabetes uncontrolled with metformin alone or with sulfonylurea: the PIONEER 3 randomized clinical trial. JAMA. 2019;321:1466-80.

33. Pieber TR, Bode B, Mertens A, et al. Efficacy and safety of oral semaglutide with flexible dose adjustment versus sitagliptin in type 2 diabetes (PIONEER 7): a multicentre, open-label, randomised, phase 3a trial. Lancet Diabetes Endocrinol. 2019;7:528-39.

34. Pratley R, Amod A, Hoff ST, et al. Oral semaglutide versus subcutaneous liraglutide and placebo in type 2 diabetes (PIONEER 4): a randomised, doubleblind, phase 3a trial. Lancet. 2019;394:39-50.

35. Moher D, Liberati A, Tetzlaff J, Altman DG, Prisma Group. Preferred reporting items for systematic reviews and meta-analyses: the PRISMA statement. BMJ. 2009;339:b2535.

36. Dias S, Welton NJ, Sutton AJ, Ades AE. NICE DSU Technical Support Document 2: a generalised linear modelling framework for pairwise and network meta-analysis of randomised controlled trials; 2011 [updated April 2014]. http://www.nicedsu.org.uk/ TSD2\%20General\%20meta\%20analysis\%20corrected \%2015April2014.pdf Accessed 14 Aug 2019.

37. Hoaglin DC, Hawkins N, Jansen JP, et al. Conducting indirect-treatment-comparison and network-meta-analysis studies: report of the ISPOR Task Force on Indirect Treatment Comparisons Good Research Practices: part 2. Value Health. 2011;14:429-37.

38. Higgins J, Green S. Cochrane Handbook for Systematic Reviews of Interventions Version 5.0.2; 2009 [updated September 2009]. www.cochranehandbook.org. Accessed 14 Aug 2019.

39. Dias S, Welton NJ, Sutton AJ, Caldwell DM, Lu G, Ades AE. NICE DSU Technical Support Document 4: inconsistency in networks of evidence based on randomised controlled trials; 2011 [updated April 2014]. http://nicedsu.org.uk/wp-content/uploads/
2016/03/TSD4-Inconsistency.final_.15April2014. pdf. Accessed 14 Aug 2019.

40. Dias S, Welton NJ, Sutton AJ, Valdwell DM, Guobing L, Ades AE. NICE DSU Technical Support Document 3: heterogeneity: subgroups, metaregression, bias and bias-adjustment; 2011. http:// www.nicedsu.org.uk. Accessed 14 Aug 2019.

41. European Medicines Agency. Albiglutide withdrawal. https://www.ema.europa.eu/en/documents/ assessment-report/public-statement-eperzan-withdra wal-marketing-authorisation-european-union_en.pdf. Accessed Jun 2019.

42. Aroda VR, Saugstrup T, Buse JB, Donsmark M, Zacho $\mathrm{J}$, Davies MJ. Incorporating and interpreting regulatory guidance on estimands in diabetes clinical trials: the PIONEER 1 randomized clinical trial as an example. Diabetes Obes Metab. 2019;21:2203-10.

43. Lunn DJ, Best N, Thomas A, Spiegelhalter D. The BUGS book: a practical introduction to Bayesian analysis. Boca Raton: CRC; 2013.

44. Welton NJ, Sutton AJ, Cooper NJ, Abrams KR, Ades AE. Evidence synthesis for decision making in healthcare. 1st ed. New York: Wiley; 2012.

45. Salanti G, Ades AE, Ioannidis JP. Graphical methods and numerical summaries for presenting results from multiple-treatment meta-analysis: an overview and tutorial. J Clin Epidemiol. 2011;64:163-71.

46. Ahren B, Johnson SL, Stewart M, et al. HARMONY 3: 104-week randomized, double-blind, placeboand active-controlled trial assessing the efficacy and safety of albiglutide compared with placebo, sitagliptin, and glimepiride in patients with type 2 diabetes taking metformin. Diabetes Care. 2014;37:2141-8.

47. Frias JP, Guja C, Hardy E, et al. Exenatide once weekly plus dapagliflozin once daily versus exenatide or dapagliflozin alone in patients with type 2 diabetes inadequately controlled with metformin monotherapy (DURATION-8): a 28 week, multicentre, double-blind, phase 3 , randomised controlled trial. Lancet Diabetes Endocrinol. 2016;4:1004-16.

48. Derosa G, Maffioli P, Salvadeo SA, et al. Exenatide versus glibenclamide in patients with diabetes. Diabetes Technol Ther. 2010;12:233-40.

49. Gallwitz B, Guzman J, Dotta F, et al. Exenatide twice daily versus glimepiride for prevention of glycaemic deterioration in patients with type 2 diabetes with metformin failure (EUREXA): an open-label, randomised controlled trial. Lancet. 2012;379:2270-8. 
50. Derosa G, Putignano P, Bossi AC, et al. Exenatide or glimepiride added to metformin on metabolic control and on insulin resistance in type 2 diabetic patients. Eur J Pharmacol. 2011;666:251-6.

51. Gallwitz B, Bohmer M, Segiet T, et al. Exenatide twice daily versus premixed insulin aspart 70/30 in metformin-treated patients with type 2 diabetes: a randomized 26-week study on glycemic control and hypoglycemia. Diabetes Care. 2011;34:604-6.

52. Zinman B, Gerich J, Buse JB, et al. Efficacy and safety of the human glucagon-like peptide-1 analog liraglutide in combination with metformin and thiazolidinedione in patients with type 2 diabetes (LEAD-4 Met + TZD). Diabetes Care. 2009;32:1224-30.

53. Buse JB, Rosenstock J, Sesti G, et al. Liraglutide once a day versus exenatide twice a day for type 2 diabetes: a 26-week randomised, parallel-group, multinational, open-label trial (LEAD-6). Lancet. 2009;374:39-47.

54. Pratley RE, Nauck M, Bailey T, et al. Liraglutide versus sitagliptin for patients with type 2 diabetes who did not have adequate glycaemic control with metformin: a 26-week, randomised, parallel-group, open-label trial. Lancet. 2010;375:1447-56.

55. Buse JB, Nauck M, Forst $T$, et al. Exenatide once weekly versus liraglutide once daily in patients with type 2 diabetes (DURATION-6): a randomised, open-label study. Lancet. 2013;381:117-24.

56. Charbonnel B, Steinberg H, Eymard E, et al. Efficacy and safety over 26 weeks of an oral treatment strategy including sitagliptin compared with an injectable treatment strategy with liraglutide in patients with type 2 diabetes mellitus inadequately controlled on metformin: a randomised clinical trial. Diabetologia. 2013;56:1503-11.

57. Bailey TS, Takacs R, Tinahones FJ, et al. Efficacy and safety of switching from sitagliptin to liraglutide in subjects with type 2 diabetes (LIRA-SWITCH): a randomized, double-blind, double-dummy, activecontrolled 26-week trial. Diabetes Obes Metab. 2016;18:1191-8.

58. Ahren B, Masmiquel L, Kumar H, et al. Efficacy and safety of once-weekly semaglutide versus once-daily sitagliptin as an add-on to metformin, thiazolidinediones, or both, in patients with type 2 diabetes (SUSTAIN 2): a 56-week, double-blind, phase $3 a$, randomised trial. Lancet Diabetes Endocrinol. 2017;5:341-54.

59. Aroda VR, Bain SC, Cariou B, et al. Efficacy and safety of once-weekly semaglutide versus once-daily insulin glargine as add-on to metformin (with or without sulfonylureas) in insulin-naive patients with type 2 diabetes (SUSTAIN 4): a randomised, open-label, parallel-group, multicentre, multinational, phase $3 a$ trial. Lancet Diabetes Endocrinol. 2017;5:355-66.

60. Dungan KM, Povedano ST, Forst T, et al. Onceweekly dulaglutide versus once-daily liraglutide in metformin-treated patients with type 2 diabetes (AWARD-6): a randomised, open-label, phase 3, non-inferiority trial. Lancet. 2014;384:1349-57.

61. Nauck M, Rizzo M, Johnson A, Bosch-Traberg H, Madsen J, Cariou B. Once-daily liraglutide versus lixisenatide as add-on to metformin in type 2 diabetes: a 26-week randomized controlled clinical trial. Diabetes Care. 2016;39:1501-9.

62. Derosa G, Franzetti IG, Querci F, et al. Exenatide plus metformin compared with metformin alone on beta-cell function in patients with type 2 diabetes. Diabet Med. 2012;29:1515-23.

63. Bergenstal RM, Wysham C, Macconell L, et al. Efficacy and safety of exenatide once weekly versus sitagliptin or pioglitazone as an adjunct to metformin for treatment of type 2 diabetes (DURATION2): a randomised trial. Lancet. 2010;376:431-9.

64. DeFronzo RA, Ratner RE, Han J, Kim DD, Fineman MS, Baron AD. Effects of exenatide (exendin-4) on glycemic control and weight over 30 weeks in metformin-treated patients with type 2 diabetes. Diabetes Care. 2005;28:1092-100.

65. Bolli GB, Munteanu M, Dotsenko S, et al. Efficacy and safety of lixisenatide once daily vs. placebo in people with type 2 diabetes insufficiently controlled on metformin (GetGoal-F1). Diabet Med. 2014;31:176-84.

66. Ahren B, Leguizamo Dimas A, Miossec P, Saubadu S, Aronson R. Efficacy and safety of lixisenatide oncedaily morning or evening injections in type 2 diabetes inadequately controlled on metformin (GetGoal-M). Diabetes Care. 2013;36:2543-50.

67. Rosenstock J, Raccah D, Koranyi L, et al. Efficacy and safety of lixisenatide once daily versus exenatide twice daily in type 2 diabetes inadequately controlled on metformin: a 24-week, randomized, open-label, active-controlled study (GetGoal-X). Diabetes Care. 2013;36:2945-51.

68. Van Gaal L, Souhami E, Zhou T, Aronson R. Efficacy and safety of the glucagon-like peptide- 1 receptor agonist lixisenatide versus the dipeptidyl peptidase4 inhibitor sitagliptin in young $(<50$ years) obese patients with type 2 diabetes mellitus. J Clin Transl Endocrinol. 2014;1:31-7.

69. Zang L, Liu Y, Geng J, et al. Efficacy and safety of liraglutide versus sitagliptin, both in combination with metformin, in Chinese patients with type 2 
diabetes: a 26-week, open-label, randomized, active comparator clinical trial. Diabetes Obes Metab. 2016;18:803-11.

70. Araki E, Inagaki N, Tanizawa Y, Oura T, Takeuchi M, Imaoka T. Efficacy and safety of once-weekly dulaglutide in combination with sulphonylurea and/or biguanide compared with once-daily insulin glargine in Japanese patients with type 2 diabetes: a randomized, open-label, phase III, non-inferiority study. Diabetes Obes Metab. 2015;17:994-1002.

71. Diamant M, Van Gaal L, Guerci B, et al. Exenatide once weekly versus insulin glargine for type 2 diabetes (DURATION-3): 3-year results of an open-label randomised trial. Lancet Diabetes Endocrinol. 2014;2:464-73.

72. Pinget M, Goldenberg R, Niemoeller E, MuehlenBartmer I, Guo H, Aronson R. Efficacy and safety of lixisenatide once daily versus placebo in type 2 diabetes insufficiently controlled on pioglitazone (GetGoal-P). Diabetes Obes Metab. 2013;15:1000-7.

73. Rosenstock J, Hanefeld M, Shamanna P, et al. Beneficial effects of once-daily lixisenatide on overall and postprandial glycemic levels without significant excess of hypoglycemia in type 2 diabetes inadequately controlled on a sulfonylurea with or without metformin (GetGoal-S). J Diabetes Complicat. 2014;28:386-92.

74. Reusch J, Stewart MW, Perkins CM, et al. Efficacy and safety of once-weekly glucagon-like peptide 1 receptor agonist albiglutide (HARMONY 1 trial): 52-week primary endpoint results from a randomized, double-blind, placebo-controlled trial in patients with type 2 diabetes mellitus not controlled on pioglitazone, with or without metformin. Diabetes Obes Metab. 2014;16:1257-64.

75. Weissman PN, Carr MC, Ye J, et al. HARMONY 4: randomised clinical trial comparing once-weekly albiglutide and insulin glargine in patients with type 2 diabetes inadequately controlled with metformin with or without sulfonylurea. Diabetologia. 2014;57:2475-84.

76. Ji L, Onishi Y, Ahn CW, et al. Efficacy and safety of exenatide once-weekly vs exenatide twice-daily in Asian patients with type 2 diabetes mellitus. J Diabetes Investig. 2013;4:53-61.

77. Liutkus J, Rosas Guzman J, Norwood P, et al. A placebo-controlled trial of exenatide twice-daily added to thiazolidinediones alone or in combination with metformin. Diabetes Obes Metab. 2010;12:1058-65.

78. Rosenstock J, Aronson R, Grumberger G, et al. Benefits of LixiLan, a titratable fixed-ratio combination of insulin glargine plus lixisenatide, versus insulin glargine and lixisenatide monocomponents in type 2 diabetes inadequately controlled on oral agents: the LixiLan-O randomized trial. Diabetes Care. 2016;39:2026-35.

79. Inagaki N, Atsumi Y, Oura T, Saito H, Imaoka T. Efficacy and safety profile of exenatide once weekly compared with insulin once daily in Japanese patients with type 2 diabetes treated with oral antidiabetes drug(s): results from a 26-week, randomized, openlabel, parallel-group, multicenter, noninferiority study. Clin Ther. 2012;34(1892-908):e1.

80. Ludvik B, Frias JP, Tinahones FJ, et al. Dulaglutide as add-on therapy to SGLT2 inhibitors in patients with inadequately controlled type 2 diabetes (AWARD-10): a 24-week, randomised, double-blind, placebo-controlled trial. Lancet Diabetes Endocrinol. 2018;6:370-81.

81. Wang W, Nevarez L, Filippova E, et al. Efficacy and safety of once-weekly dulaglutide versus insulin glargine in mainly Asian patients with type 2 diabetes mellitus on metformin and/or a sulphonylurea: a 52-week open-label, randomized phase III trial. Diabetes Obes Metab. 2019;21:234-43.

82. Wysham $\mathrm{CH}$, Rosenstock J, Vetter ML, Dong F, Ohman P, Iqbal N. Efficacy and tolerability of the new autoinjected suspension of exenatide once weekly versus exenatide twice daily in patients with type 2 diabetes. Diabetes Obes Metab. 2018;20:165-72.

83. Gadde KM, Vetter ML, Iqbal N, Hardy E, Ohman P. Efficacy and safety of autoinjected exenatide onceweekly suspension versus sitagliptin or placebo with metformin in patients with type 2 diabetes: the DURATION-NEO-2 randomized clinical study. Diabetes Obes Metab. 2017;19:979-88.

84. Frias JP, Nauck MA, Van J, et al. Efficacy and safety of LY3298176, a novel dual GIP and GLP-1 receptor agonist, in patients with type 2 diabetes: a randomised, placebo-controlled and active comparatorcontrolled phase 2 trial. Lancet. 2018;392:2180-93.

85. Yu Pan C, Han P, Liu X, et al. Lixisenatide treatment improves glycaemic control in Asian patients with type 2 diabetes mellitus inadequately controlled on metformin with or without sulfonylurea: a randomized, double-blind, placebo-controlled, 24-week trial (GetGoal-M-Asia). Diabetes Metab Res Rev. 2014;30:726-35.

86. Ahmann AJ, Capehorn M, Charpentier G, et al. Efficacy and safety of once-weekly semaglutide versus exenatide ER in subjects with type 2 diabetes (SUSTAIN 3): a 56-week, open-label, randomized clinical trial. Diabetes Care. 2018;41:258-66. 
87. Novo Nordisk. Data on file: results meetingsemaglutide subcutaneous vs. dulaglutide (SUSTAIN 7). 2017.

88. Sun F, Chai S, Yu K, et al. Gastrointestinal adverse events of glucagon-like peptide-1 receptor agonists in patients with type 2 diabetes: a systematic review and network meta-analysis. Diabetes Technol Ther. $2015 ; 17: 35-42$.

89. Sikirica MV, Martin AA, Wood R, Leith A, Piercy J, Higgins V. Reasons for discontinuation of GLP1 receptor agonists: data from a real-world cross-sectional survey of physicians and their patients with type 2 diabetes. Diabetes Metab Syndr Obes. 2017;10:403-12.

90. Zaccardi F, Htike ZZ, Webb DR, Khunti K, Davies MJ. Benefits and harms of once-weekly glucagonlike peptide- 1 receptor agonist treatments: a systematic review and network meta-analysis. Ann Intern Med. 2016;164:102-13.

91. Kayaniyil S, Lozano-Ortega G, Bennett HA, et al. A network meta-analysis comparing exenatide once weekly with other GLP-1 receptor agonists for the treatment of type 2 diabetes mellitus. Diabetes Ther. 2016;7:27-43.
92. Orme ME, Nguyen H, Lu JY, Thomas SA. Comparative effectiveness of glycemic control in patients with type 2 diabetes treated with GLP-1 receptor agonists: a network meta-analysis of placebo-controlled and active-comparator trials. Diabetes Metab Syndr Obes. 2017;10:111-22.

93. Witkowski M, Wilkinson L, Webb N, Weids A, Glah D, Vrazic H. A systematic literature review and network meta-analysis comparing once-weekly semaglutide with other GLP-1 receptor agonists in patients with type 2 diabetes previously receiving 1-2 oral anti-diabetic drugs. Diabetes Ther. 2018;9:1149-67.

94. Barnett $\mathrm{AH}$, Orme ME, Fenici P, Townsend R, Wygant G, Roudaut M. Systematic review and network meta-analysis to compare dapagliflozin with other diabetes medications in combination with metformin for adults with type 2 diabetes. Diabetes Intern Med. 2014;S6:006.

95. Turner RM, Spiegelhalter DJ, Smith GC, Thompson SG. Bias modelling in evidence synthesis. J R Stat Soc Ser A Stat Soc. 2009;172:21-47. 